\title{
Contemporary treatment patterns in plaque psoriasis and psoriatic arthritis
}

\author{
Dorota Jenerowicz ${ }^{1}$, Justyna Kaznowska ${ }^{1}$, Paweł Bartkiewicz ${ }^{1}$, Anna Sadowska-Przytocka1, Marcin Szymkowiak², \\ Zygmunt Adamski ${ }^{1}$, Magdalena Czarnecka-Operacz ${ }^{1}$
}

${ }^{1}$ Department of Dermatology, Poznan University of Medical Sciences, Poznan, Poland ${ }^{2}$ Department of Statistics, Poznan University of Economics and Business, Poznan, Poland

Adv Dermatol Allergol 2021; XXXVIII (1): 80-84

DOI: https://doi.org/10.5114/ada.2019.91502

\begin{abstract}
Introduction: Psoriasis is a chronic inflammatory skin disease affecting about $2 \%$ of the general population. Although there are many treatment options, and new medications have been introduced, the disease is considered not curable, and it may seriously affect patients' quality of life.

Aim: The authors present contemporary treatment patterns used by dermatologists in Poland to manage plaque psoriasis and psoriatic arthritis, particularly regarding systemic treatment. The authors also aimed to analyse how these treatment patterns are influenced by the guidelines of the Polish Dermatological Society.

Material and methods: The author's questionnaire, consisting of 13 questions was used. It included demographic and professional characteristics of questioned dermatologists, as well as the assessment of the attitudes towards management of plaque psoriasis and psoriatic arthritis.

Results: A total of 132 dermatologists completed the questionnaire. Most of the specialists worked in out-patient clinics and private practices. The most commonly used topicals for psoriasis included: glucocorticosteroids, a combination of glucocorticosteroid and vitamin D analogue and salicylic acid. Regarding the treatment of psoriatic arthritis, most of the specialists declared using systemic therapy and a combination of systemic therapy and phototherapy. The majority of the respondents were particularly concerned with possible side effects or difficulties in qualifying and monitoring the patients, and less frequently on the cost of the therapy.

Conclusions: Observations suggest that $60 \%$ of physicians have some reservation to initiate systemic treatment in outpatient clinics, and they admit that they lack additional training. On the other hand, it seems also that the organization of systemic treatment in psoriasis may generate these difficulties and thus necessitate additional effort. Another factor could be the budget - not only regarding healthcare professionals, but also the patient, sometimes financing various investigations from private resources.
\end{abstract}

Key words: treatment, psoriasis, dermatologist.

\section{Introduction}

Psoriasis is a chronic inflammatory skin disease affecting about $2 \%$ of the general population. Although there are many treatment options, the disease is not curable, and it may seriously affect patients' quality of life. The development of new drugs for psoriasis does not necessarily cause a change in the quality of care given to patients. New discoveries frequently take a long time to arrive in daily clinical practice. High-quality, evidencebased guidelines can make an important contribution to improving medical care $[1,2]$.
To meet the expectations of Polish dermatologists, in 2014 the Polish Dermatological Society introduced guidelines for the treatment of moderate to severe psoriasis [2], which were a continuation of the guidelines for diagnostics and treatment of mild psoriasis and childhood psoriasis published in 2012 [3]. In order to reach the majority of Polish dermatologists, the guidelines have been implemented in numerous ways, i.e. publication in Polish Dermatology Review with online access, and presentations at national and local meetings of the Polish Dermatological Society.

Address for correspondence: Dorota Jenerowicz, Department of Dermatology, Poznan University of Medical Sciences, 49 Przybyszewskiego St, Poznan, Poland, e-mail: djenerowicz@yahoo.com

Received: 5.03.2019, accepted: 15.07.2019. 


\section{Aim}

The aim of our study was to assess treatment patterns used by Polish dermatologists in the management of plaque psoriasis and psoriatic arthritis, with special attention to systemic treatment, and also how these treatment patterns are compatible with the guidelines of the Polish Dermatological Society [2, 3].

\section{Material and methods}

The questionnaire consisted of 13 questions, assessing the demographic and professional characteristics of the dermatologists: type of practice, scientific title, and years in practice. The second section assessed the attitudes towards management of plaque psoriasis and psoriatic arthritis: the use of topical drugs, systemic treatment, and their combination. The specialists were also asked if an out-patient introduction of systemic treatment causes any doubts or reservations and the nature of the latter.

\section{Results}

A total of 132 dermatologists completed the questionnaire. Physician and practice demographics are summarised in Table 1. Most of the specialists worked in out-patient clinics and private practices. Eighteen percent of physicians were employed in outpatient clinics within University Hospital or other hospital. Over $70 \%$ of questioned specialists were MDs, whereas less than 30\% had a PhD title. Almost $70 \%$ of doctors declared at least 15 years of practice.

Doctors said they could treat psoriasis with combined therapy: systemic and topical (49\%) plus phototherapy and topical (39\%). Oral methotrexate (55\%), retinoids (49\%), and UVB radiation (38\%) were among the most frequently used systemic regimens. The most commonly used topicals included: glucocorticosteroids (95\%), a combination of glucocorticosteroid and vitamin D analogue (85\%), and salicylic acid (76\%).
Regarding the treatment of psoriatic arthritis, most of the specialists declared using systemic therapy and a combination of systemic therapy and phototherapy. Methotrexate in its oral form was the most frequently used systemic treatment in psoriatic arthritis. The subcutaneous form of methotrexate was declared to be used less often (42\%). Sixteen percent of the respondents pointed to the use of biologicals and $12 \%$ to the use of cyclosporine A.

The majority of the respondents were particularly concerned with possible side effects or difficulties in qualifying and monitoring the patients, and less frequently on the cost of the therapy. Vast majority of respondents claimed that their decision on the systemic management of plaque psoriasis was mainly influenced by the lack of training/courses.

Another part of the study included the analysis of potential statistically significant associations between the

Table 1. Demographics and professional characteristics of interviewed dermatologists

\begin{tabular}{ll}
\hline $\begin{array}{l}\text { Demographics and professional } \\
\text { characteristics }\end{array}$ & Percent \\
\hline Practice type: & 72 \\
\hline Outpatient clinic & 53 \\
\hline Private practice & 18 \\
\hline Hospital & 71.2 \\
\hline Scientific title: & 28 \\
\hline Medical doctor (MD) & 0.8 \\
\hline PhD & 15.3 \\
\hline Ass. Prof./Prof. & 14.5 \\
\hline Professional experience: & 31.3 \\
\hline 5-10 years & 38.9 \\
\hline 11-15 years & \\
\hline $16-20$ years & \\
\hline More than 20 years & \\
\hline
\end{tabular}

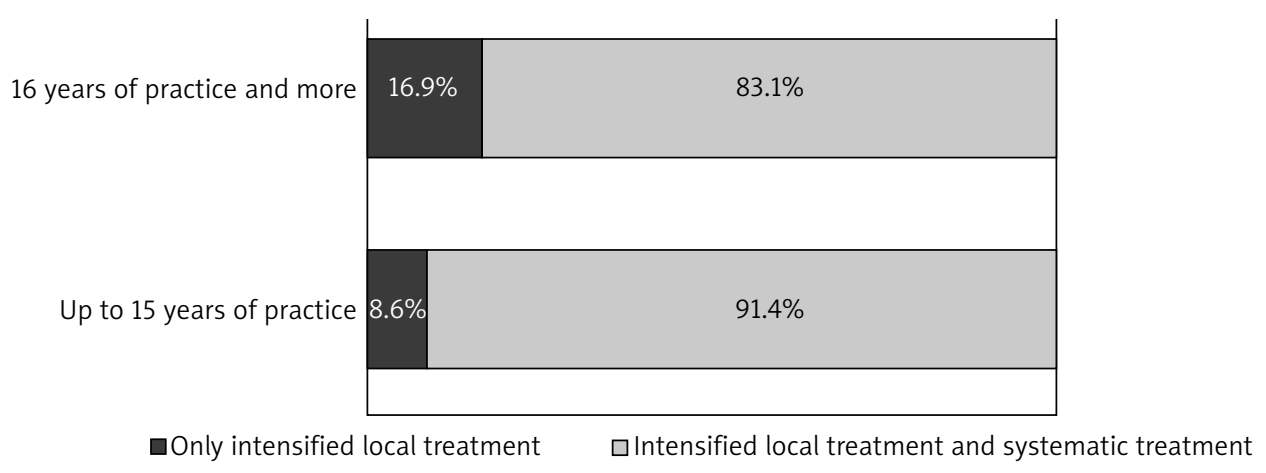

Figure 1. Psoriasis treatment trends and doctors' years of practice 
mode of treatment of patients with moderate to severe plaque psoriasis and the doctors' experience, workplace, and title/grade. For the needs of the analysis, Fisher's exact test was used, by means of which dependencies in contingency tables containing small numbers in some cells could be detected. The application of this test resulted from the fact that 132 physicians took part in the whole questionnaire, which, with the necessity of constructing appropriate contingency tables for variables containing at least two variants, meant that in some cells of such a table the abundance did not exceed five. The level of significance was assumed as $\alpha=0.05$. Statistical analysis was performed using the R software.

First, we analysed whether there was a statistically significant association between the way patients are treated and the work experience (Figure 1). The $p$-value determined with the use of Fisher's exact test was 0.3892, indicating no dependency between the analysed variables.

Regardless of the length of experience in medicine, the vast majority of physicians used two forms of treatment, i.e. intensified local treatment and systemic treatment (Figure 2). The percentage of doctors using only intensified local treatment was slightly higher in the group of subjects being in practice 16 years or more (nearly
$17 \%)$ than in the group of doctors with an internship of up to 15 years (nearly 9\%). However, these were not significant associations in the statistical sense.

A similar analysis was performed between the title/ grade of the physician and the treatment. With regard to the first variable, two categories were distinguished: medical doctor or PhD as well as associated professor or professor of medical sciences. The $p$-value determined in the exact Fisher test was 0.998, meaning there was no relationship between the two variables analysed (Figure 2).

The vast majority of physicians, regardless of the title or the academic degree, treated moderate to severe plaque psoriasis, using both intensified local and systemic treatment. In the group of medical doctors as well as those with PhDs (associated professors) the frequency of usage of only intensified local treatment was low (12-15\%).

The study also included the relationship between the treatment of psoriasis and the workplace. At the same time, three categories were distinguished because of small numbers: private practice only, outpatient clinic at the University Hospital or outpatient dermatology clinic only and outpatient clinic at the University Hospital or outpatient dermatology clinic plus private practice. The determined $p$-value of 0.1075 indicates the lack of

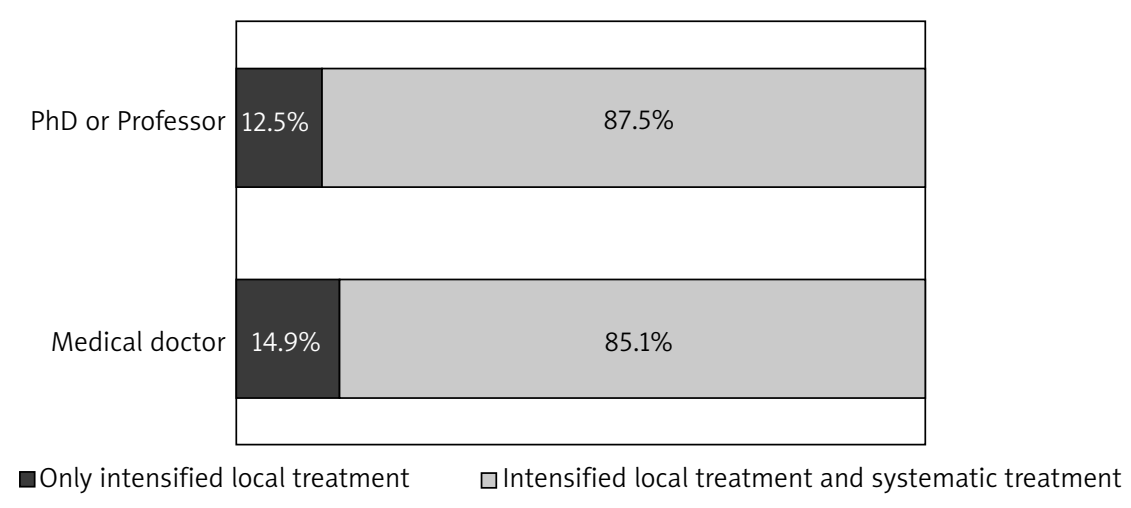

Figure 2. Psoriasis mode of treatment and doctors' grade/scientific title

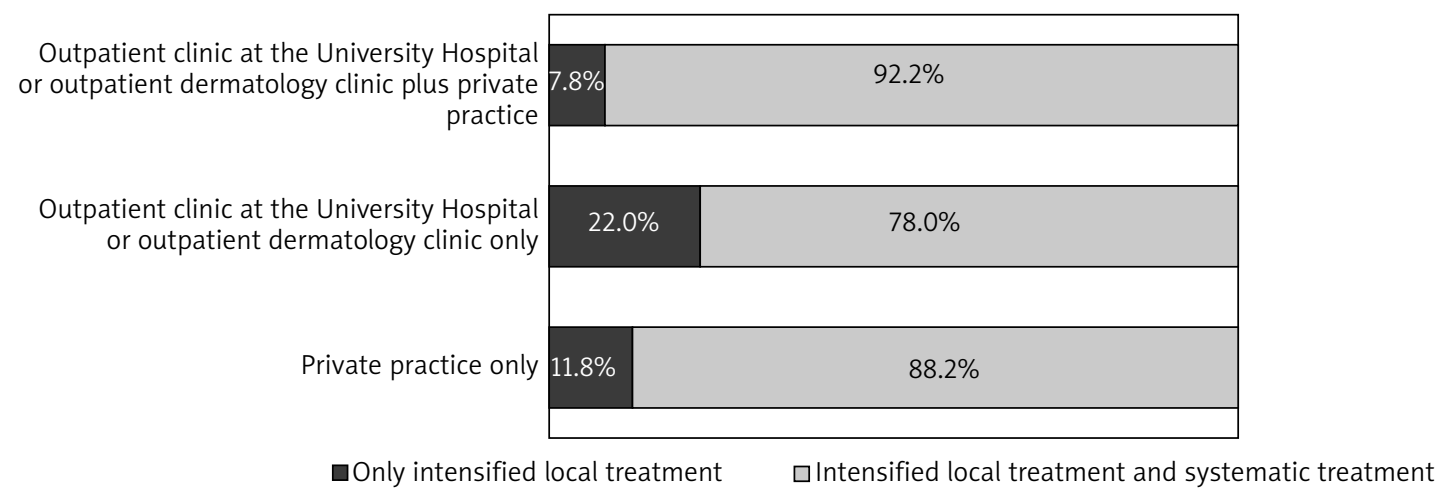

Figure 3. Psoriasis treatment trends and doctors' place of practice 
a statistically significant relationship between the place of work and the mode of psoriasis. The vast majority of doctors, regardless of the place of their practice, applied both intensified local treatment and systemic treatment. The percentage of those who only used intensified local treatment was generally low $-11.8 \%, 22 \%$, and $7.8 \%$, respectively (in the categories indicated above - Figure 3).

\section{Discussion}

Despite the undeniable progress that has been made in medicine and pharmacology, the treatment of psoriasis and psoriatic arthritis remains a challenge. There is no ideal antipsoriatic agent; however, because we consider both patients' and doctors' points of view, an administered drug should be effective as monotherapy and have a rapid onset of action. It should also be characterised by the low risk of organ toxicity, malignancy, and infection, and patients of all ages should benefit from its use [4]. Although there is currently no definite cure for psoriasis, antipsoriatic medications are expected to gain continuous effective control of the disease, and their cost should be affordable to most patients and to the national health services.

According to our knowledge, this is the first study investigating treatment patterns of plaque psoriasis and psoriasis arthritis in Poland. We interviewed 132 dermatologists out of 2555 active Polish representatives of this specialty. Systemic treatment, with reference to Polish psoriasis guidelines, is indicated in cases of moderate to severe psoriasis (PASI greater than 10, BSA greater than 10) if the disease has a severe impact on quality of life $(D L Q I \geq 10)$ or in the case of patients with an active psoriatic arthritis. The use of biologics is also recommended for patients with moderate to severe psoriasis, but only if other systemic drugs have not been effective, or if they are not tolerated or are contraindicated.

The vast majority of participating dermatologists (85\%) claimed to use both systemic and topical treatment in patients with moderate to severe psoriasis, which seems to be an appropriate ratio. According to our observations, neither the doctors' seniority and workplace, nor the doctors' scientific title and grade significantly influenced the mode of moderate to severe plaque psoriasis treatment. The most frequently prescribed drug was methotrexate. Methotrexate has remained the treatment of choice for many patients since its first use over half a century ago, due to its high efficacy, low cost, relatively easy administration, and efficacy in concomitant psoriatic arthritis [5, 6]. Without doubt patients have to be selected carefully and monitored regularly, particularly with respect to liver and bone marrow toxicity, because this helps to reduce severe side-effects even during longterm treatment with methotrexate [5]. The second most frequently prescribed agent was acitretin, which was applied in preference to cyclosporine. Only half of the responders ordered cyclosporine; however, it is a wellknown indicated first-line therapy for women of reproductive age $[2,7]$.

The introduction of biological drugs became the greatest advance in the management of moderate to severe psoriasis of the past decade. It should be emphasised that biologicals have significantly changed psoriasis treatment by selectively targeting immune signalling molecules involved in psoriasis pathogenesis [8].

However, the use of biologics is still not widely available in Poland. According to Rencz et al. [9], on average $0.25 \%$ of all psoriasis patients, or five psoriasis patients per 100,000 population are treated with biologics in Central and Eastern Europe (Poland - 0.04\%). According to the authors, the main drawbacks influencing the use of biologic drugs include financing and coverage eligibility criteria. The high baseline PASI and DLQI scores required in Bulgaria, Croatia, and in Poland largely explain the lower use observed in these countries. Therefore, it seems that the use of biologics as a cure for psoriasis in Poland might not be hampered by the high costs of these medications, but is rather influenced by disadvantageous coverage policies and guidelines.

Our survey has shown that $60 \%$ of physicians had some reservations regarding initiation of systemic treatment in outpatient clinics. Among these respondents, the majority had some concerns, mainly about side effects, but also about indications and monitoring therapy. These dermatologists declared that they were lacking training. Similarly to Nast et al. [10], it is reasonable to conclude that these uncertainties lead to underuse of systemic therapy. It is, however, worth emphasising that Polish guidelines include detailed data on recommendations, contraindications, and diagnostic procedures before initiating and also during treatment, as well as possible side effects. According to our observations, at least five large dermatological conferences take place in Poland every year, and even more local meetings are available with psoriasis discussed as a frequent subject. It seems that there are lot of opportunities to train and ask questions in case of any doubts. Hence, perhaps it is not only the need for training but also the whole organisation of systemic treatment that influences all the difficulties and necessitates additional effort. We have to remember that initiation of systemic treatment is time consuming and requires thorough knowledge of, and experience with, the different treatment options. It also demands more comprehensive patient history taking and exclusion of chronic infections or malignancies. It may also affect another important factor: the budget - not only of the doctor but also of the patient, with the latter often financing lab tests and other investigations from private resources.

It is acknowledged that this study has some limitations. The questionnaire is the simplest and cheapest way of collecting information on treatment patterns, but it is not accurate. Dermatologists were supposed to point 
out the methods of treatment they use, not the percentage of patients they treat with systemic drugs. To provide reliable data on the percentage of patients with moderate to severe psoriasis, who are undergoing systemic treatment, we should have an insight into the patients' history because neither a general medical database nor electronic prescriptions are available in Poland.

When it comes to the treatment of psoriasis arthritis, most of the dermatologists declared using systemic therapy and a combination of systemic therapy and phototherapy. The most frequently used drugs were methotrexate, followed by biologicals and cyclosporine A. However, not all participants responded to this question; $11 \%$ abstained from answering. The guidelines created by the Polish Dermatological Society do not include treatment of psoriasis arthritis because it is the domain of rheumatologists, and dermatologists may not feel confident or have enough experience in this field. As a heterogeneous disease, psoriasis arthritis should be evaluated and managed by a multidisciplinary care unit, not by a dermatologist and rheumatologist separately. A retrospective observational study performed by Urruticoechea-Arana et al. [11] showed that close collaboration between dermatologists and rheumatologists with experience and interest in psoriatic disease facilitates a proper evaluation of the skin and joint condition, subsequently resulting in an earlier diagnosis of psoriasis arthritis and comorbidities, leading to a more comprehensive treatment approach, and finally improving patient satisfaction.

In 2008 Nast et al. [12] designed a study in which private-practice dermatologists were asked to provide continuous documentation from every visit by patients with psoriasis vulgaris. The documentation form included, among others: disease activity and treatment choices. It is important to mention that participating dermatologists were offered $€ 250$ reimbursement. In total, 54 dermatologists engaged in this study, and 4797 patient visits were documented. For patients with moderate to severe psoriasis, systemic treatment was prescribed in $31 \%$, and in $17 \%$ of cases patient received a combination of phototherapy and topical treatment. It was an example of a well-designed study that provided reliable data on treatment pattern of psoriasis. We are also considering a similar study in the future, providing more precise date; however, it would require a sponsor that would cover the expenses.

\section{Conflict of interest}

The authors declare no conflict of interest.

\section{References}

1. Feldmann SR, Goffe B, Rice G, et al. The challenge of managing psoriasis: unmet medical needs and stakeholder perspectives. Am Health Drug Benefits 2016; 9: 504-13.

2. Szepietowski J, Adamski Z, Chodorowska G, et al. Treatment of psoriasis vulgaris: guidelines of the Polish Dermatological
Society. Part II: moderate to severe psoriasis. Przegl Dermatol 2014; 101: 455-72.

3. Szepietowski J, Adamski Z, Chodorowska G, et al. Diagnostics and treatment of psoriasis vulgaris: guidelines of the Polish Dermatological Society. Part I: mild psoriasis, psoriasis in children. Przegl Dermatol 2012; 99: 83-96.

4. Ferrándiz C, Carrascosa JM, Boada A. A new era in the management of psoriasis? The biologics: facts and controversies. Clin Dermatol 2010; 28: 81-7.

5. Haustein UF, Rytter M. Methotrexate in psoriasis: 26 years' experience with low-dose long-term treatment. J Eur Acad Dermatol Venereol 2000; 14: 382-8.

6. Kalb RE, Strober B, Weinstein G, et al. Methotrexate and psoriasis: 2009 National Psoriasis Foundation Consensus Conference. J Am Acad Dermatol 2009; 60: 824-37.

7. Strober BE. Methotrexate and cyclosporine in psoriasis revisited. Semin Cutan Med Surg 2014; 33: S27-30.

8. Cline A, Hill D, Lewallen R, et al. Current status and future prospects for biologic treatments of psoriasis. Expert Rev Clin Immunol 2016; 12: 1273-87.

9. Rencz F, Kemény L, Gajdácsi JZ, et al. Use of biologics for psoriasis in Central and Eastern European countries. J Eur Acad Dermatol Venereol 2015; 29: 2222-30.

10. Nast A, Reytan N, Rosumeck S, et al. Low prescription rate for systemic treatments in the management of severe psoriasis vulgaris and psoriatic arthritis in dermatological practices in Berlin and Brandenburg, Germany: results from a patient registry. J Eur Acad Dermatol Venereol 2008; 22: 1337-42.

11. Urruticoechea-Arana A, Serra Torres M, Hergueta Diaz M, et al. Experience and satisfaction with a multidisciplinary care unit for patients with Psoriasis and psoriatic arthritis. Reumatol Clin 2019; 15: 237-41.

12. Nast A, Erdmann R, Hofelich $V$, et al. Do guidelines change the way we treat? Studying prescription behaviour among private practitioners before and after the publication of the German Psoriasis Guidelines. Arch Dermatol Res 2009; 301: 553-9. 